\title{
A SUMMABILITY TYPE FACTOR THEOREM
}

\author{
B. E. RHOADES AND EKREM SAVAS
}

\begin{abstract}
We obtain sufficient conditions for the series $\sum a_{n} \epsilon_{n}$ to be absolutely summable of order $k$ by a wieghted mean method.
\end{abstract}

The concept of absolute summability of order $k$ was defined by Flett [2] as follows. Let $\sum a_{n}$ be a given infinite series with partial sums $s_{n}$, and let $\sigma_{n}^{\alpha}$ denote the $n$-th Cesáro means of order $\alpha, \alpha>-1$, of the sequence $\left\{s_{n}\right\}$. The series $\sum a_{n}$ is said to be summable $|C, \alpha|_{k}, k \geq 1, \alpha>-1$ if

$$
\sum_{n=1}^{\infty} n^{k-1}\left|\Delta \sigma_{n-1}^{\alpha}\right|^{k}<\infty,
$$

where, for any sequence $\left\{b_{n}\right\}, \Delta b_{n}=b_{n}-b_{n+1}$.

In defining absolute summability of order $k$ for weighted mean methods Bor [1] and others used the definition

$$
\sum_{n=1}^{\infty}\left(\frac{P_{n}}{p_{n}}\right)^{k-1}\left|\Delta u_{n-1}\right|^{k}<\infty
$$

where

$$
u_{n}:=\sum_{\nu=0}^{n} p_{\nu} s_{\nu} .
$$

In using (2) as the definition, it was apparently assumed that the $n$ in (1) represented the reciprocal of the $n$th main diagonal term of $(C, 1)$. But this interpretation cannot be correct. For, if it were, then the Cesáro methods $(C, \alpha)$, for $\alpha \neq 1$ would have to satisfy the condition

$$
\sum_{n=1}^{\infty}\left(n^{\alpha}\right)^{k-1}\left|\Delta \sigma_{n-1}^{\alpha}\right|^{k}<\infty .
$$

However, Flett [2] stays with $n$ for all values of $\alpha>-1$.

In a recent paper [3], Sulaiman proved the following two results.

Received September 5, 2002.

2000 Mathematics Subject Classification. Primary: 40G99; Secondary: 40G05, $40 \mathrm{D} 15$.

Key words and phrases. Absolute summability, weighted mean matrix, Cesáro matrix, summability factor. 
Theorem 1. (A) Let $\left\{p_{n}\right\}$ be a sequence of positive numbers. Let $T_{n}$ be the $\left(\bar{N}, p_{n}\right)$ mean of the series $\sum a_{n}$. If

$$
\begin{aligned}
& \sum_{n=1}^{\infty} n^{k-1}\left|\epsilon_{n}\right|^{k}\left|\Delta T_{n-1}\right|^{k}<\infty \\
& \sum_{n=1}^{\infty} n^{k-k \alpha-1}\left(\frac{P_{n}}{p_{n}}\right)^{k}\left|\epsilon_{n}\right|^{k}\left|\Delta T_{n-1}\right|^{k}<\infty, \quad(0<\alpha<1) \\
& \sum_{n=1}^{\infty} n^{-1}\left(\frac{P_{n}}{p_{n}}\right)^{k}\left|\epsilon_{n}\right|^{k}\left|\Delta T_{n-1}\right|^{k}<\infty, \quad(\alpha \geq 1) \\
& \sum_{n=1}^{\infty} n^{-1}\left(\frac{P_{n}}{p_{n}}\right)^{k}\left|\Delta \epsilon_{n}\right|^{k}\left|\Delta T_{n-1}\right|^{k}<\infty
\end{aligned}
$$

then the series $\sum a_{n} \epsilon_{n}$ is summable $|C, \alpha|_{k}, k \geq 1, \alpha>0$.

(B) Let $\left\{p_{n}\right\}$ be a sequence of positive numbers satisfying

$$
\begin{array}{ll}
\text { (i) } & n p_{n}=O\left(P_{n}\right) \\
\text { (ii) } & P_{n}=O\left(n p_{n}\right) .
\end{array}
$$

Let $\left\{\lambda_{n}\right\},\left\{\epsilon_{n}\right\}$ be such that $\left\{\lambda_{n}\right\}$ is nonnegative, nondecreasing, $n^{1-\alpha} \lambda_{n}\left|\epsilon_{n}\right|=O(1)$ for $0<\alpha<1, \lambda_{n}\left|\epsilon_{n}\right|=O(1), \epsilon_{n}=o(1)$ for $\alpha \geq 1, \Delta \epsilon_{n}=O\left(n^{-1}\left|\epsilon_{n}\right|\right)$, and

$$
\sum_{n=1}^{m}\left(\frac{P_{n}}{p_{n}}\right)^{k-1}\left|\Delta T_{n-1}\right|^{k}=O\left(\lambda_{m}^{k}\right), \quad m \rightarrow \infty .
$$

Then, in order to have the series $\sum a_{n} \epsilon_{n}$ summable $|C, \alpha|_{k}$, it is sufficient that

$$
\sum_{n=1}^{\infty} n^{2-\alpha} \lambda_{n}\left|\Delta^{2} \epsilon_{n}\right|<\infty, \quad(0<\alpha<1)
$$

and

$$
\sum_{n=1}^{\infty} n \lambda_{n}\left|\Delta^{2} \epsilon_{n}\right|<\infty, \quad(\alpha \geq 1)
$$

Theorem 2. (A) Let $\left\{p_{n}\right\}$ be a sequence of positive numbers. Let $t_{n}^{1}$ be the nth $(C, 1)$-mean of the sequence $\left\{n a_{n}\right\}$. If

$$
\begin{aligned}
& \sum_{n=1}^{\infty} \frac{p_{n}}{P_{n}}\left|\epsilon_{n}\right|^{k}\left|t_{n}^{1}\right|^{k}<\infty \\
& \sum_{n=1}^{\infty} \frac{1}{n^{k}}\left(\frac{P_{n}}{p_{n}}\right)^{k-1}\left|\epsilon_{n}\right|^{k}\left|t_{n}^{1}\right|^{k}<\infty \\
& \sum_{n=1}^{\infty}\left(\frac{P_{n}}{p_{n}}\right)^{k-1}\left|\Delta \epsilon_{n}\right|^{k}\left|t_{n}^{1}\right|^{k}<\infty,
\end{aligned}
$$


then the series $\sum a_{n} \epsilon_{n}$ is summable $\left|\bar{N}, p_{n}\right|_{k}, k \geq 1$.

(B) Let $\left\{p_{n}\right\}$ be a sequence of positive numbers such that (3) holds. Let $\left\{\lambda_{n}\right\},\left\{\epsilon_{n}\right\}$ be such that $\left\{\lambda_{n}\right\}$ is nonnegative, nondecreasing, $\lambda_{n}\left|\epsilon_{n}\right|=O(1), \epsilon_{n}=o(1), \Delta \epsilon_{n}=$ $O\left(n^{-1}\left|\epsilon_{n}\right|\right)$, and

$$
\sum_{n=1}^{m} n^{-1}\left|t_{n}^{1}\right|^{k}=O\left(\lambda_{n}^{k}\right)
$$

Then, in order to have the series $\sum a_{n} \epsilon_{n}$ summable $\left|\bar{N}, p_{n}\right|_{k}, k \geq 1$, it is sufficient that

$$
\sum_{n=1}^{\infty} n \lambda_{n}\left|\Delta^{2} \epsilon_{n}\right|<\infty
$$

In the proof of Theorem 1, Sulaiman uses the correct definition for absolute Cesáro summability. However, in proving Theorem 2, he reverts to (2) for absolute weighted mean summability.

In this paper we shall prove the corresponding version of Theorem 2, using the correct definition (1).

Theorem 3. (A) Let $\left\{p_{n}\right\}$ be a positive sequence such that

$$
\sum_{n=\nu+1}^{\infty} \frac{n^{k-1}}{P_{n-1}}\left(\frac{p_{n}}{P_{n}}\right)^{k}=O\left(\frac{\nu^{k-1} p_{\nu}^{k-1}}{P_{\nu}^{k}}\right)
$$

Let $t_{n}^{1}$ denote the nth $(C, 1)$ mean of $\left\{n a_{n}\right\}$. If

$$
\begin{aligned}
& \sum_{\nu=1}^{\infty} \nu^{k-1}\left|\epsilon_{\nu}\right|^{k}\left|t_{\nu}^{1}\right|^{k}<\infty \\
& \sum_{\nu=1}^{\infty} \nu^{k-1}\left|\Delta \epsilon_{\nu}\right|^{k}\left|t_{\nu}^{1}\right|^{k}<\infty
\end{aligned}
$$

then the series $\sum a_{n} \epsilon_{n}$ is summable $\left|\bar{N}, p_{n}\right|_{k}, k \geq 1$.

(B) Let $\left\{p_{n}\right\}$ be a positive sequence satisfying (3). Let $\left\{\lambda_{n}\right\},\left\{\epsilon_{n}\right\}$ be such that $\left\{\lambda_{n}\right\}$ is nonnegative, nondecreasing, $n \lambda_{n}\left|\epsilon_{n}\right|=O(1), \epsilon_{n}=o(1), \Delta \epsilon_{n}=O\left(n^{-1}\left|\epsilon_{n}\right|\right)$ and

$$
\sum_{\nu=1}^{n} \nu^{-1}\left|t_{n}^{1}\right|^{k}=O\left(\lambda_{n}^{k}\right)
$$

Then, in order to have $\sum a_{n} \epsilon_{n}$ summable $\left|\bar{N}, p_{n}\right|_{k}, k \geq 1$, it is sufficient that

$$
\sum_{\nu=1}^{\infty} \nu^{2} \lambda_{\nu}\left|\Delta^{2} \epsilon_{\nu}\right|<\infty
$$


Proof. Part (A). Let $Q_{n}$ denote the $\left(\bar{N}, p_{n}\right)$-mean of the series $\sum a_{n} \epsilon_{n}$. Then, as in $[3]$

$$
\begin{aligned}
Q_{n} & =\frac{1}{P_{n}} \sum_{\nu=0}^{n} p_{\nu} \sum_{r=0}^{\nu} a_{r} \epsilon_{r}=\frac{1}{P_{n}} \sum_{\nu=0}^{n}\left(P_{n}-P_{\nu-1}\right) a_{\nu} \epsilon_{\nu} . \\
Q_{n}-Q_{n-1} & =\frac{p_{n}}{P_{n} P_{n-1}} \sum_{\nu=1}^{n} P_{\nu-1} a_{\nu} \epsilon_{\nu} \\
& =\frac{p_{n}}{P_{n} P_{n-1}} \sum_{\nu=1}^{n} \nu a_{\nu} \frac{P_{\nu-1} \epsilon_{\nu}}{\nu} \\
& =\frac{p_{n}}{P_{n} P_{n-1}}\left[\sum_{\nu=1}^{n-1}(\nu+1) t_{\nu}^{1}\left\{-\frac{p_{\nu} \epsilon_{\nu}}{\nu}+\frac{P_{\nu} \epsilon_{\nu}}{\nu(\nu+1)}+\frac{P_{\nu} \Delta \epsilon_{\nu}}{\nu+1}\right\}+\frac{n+1}{n} P_{n-1} \epsilon_{n} t_{n}^{1}\right] \\
& =Q_{n 1}+Q_{n 2}+Q_{n 3}+Q_{n 4} .
\end{aligned}
$$

Using Hölder's inequality and (5),

$$
\begin{aligned}
\sum_{n=2}^{m+1} n^{k-1}\left|Q_{n 1}\right|^{k} & =\sum_{n=2}^{m+1} n^{k-1}\left|\frac{p_{n}}{P_{n} P_{n-1}} \sum_{\nu=1}^{n-1}\left(1+\frac{1}{\nu}\right) p_{\nu} \epsilon_{\nu} t_{\nu}^{1}\right|^{k} \\
& =O(1) \sum_{n=2}^{m+1} \frac{n^{k-1}}{P_{n-1}}\left(\frac{p_{n}}{P_{n}}\right)^{k}\left[\sum_{\nu=1}^{n-1} p_{\nu}\left|\epsilon_{\nu}\right|^{k}\left|t_{\nu}^{1}\right|^{k}\right]\left[\frac{1}{P_{n-1}} \sum_{\nu=1}^{n-1} p_{\nu}\right]^{k-1} \\
& =O(1) \sum_{n=2}^{m+1} \frac{n^{k-1}}{P_{n-1}}\left(\frac{p_{n}}{P_{n}}\right)^{k} \sum_{\nu=1}^{n-1} p_{\nu}\left|\epsilon_{\nu}\right|^{k}\left|t_{\nu}^{1}\right|^{k} \\
& =O(1) \sum_{\nu=1}^{m} p_{\nu}\left|\epsilon_{\nu}\right|^{k}\left|t_{\nu}^{1}\right|^{k} \sum_{n=\nu+1}^{m+1} \frac{n^{k-1}}{P_{n-1}}\left(\frac{p_{n}}{P_{n}}\right)^{k} \\
& =O(1) \sum_{\nu=1}^{m} p_{\nu}\left|\epsilon_{\nu}\right|^{k}\left|t_{\nu}^{1}\right|^{k} \frac{\nu^{k-1} p_{\nu}^{k-1}}{P_{\nu}^{k}} \\
& =O(1) \sum_{\nu=1}^{m} \nu^{k-1}\left(\frac{p_{\nu}}{P_{\nu}}\right)^{k}\left|\epsilon_{\nu}\right|^{k}\left|t_{\nu}^{1}\right|^{k} \\
& \leq O(1) \sum_{\nu=1}^{m} \nu^{k-1}\left|\epsilon_{\nu}\right|^{k}\left|t_{n}^{1}\right|^{k}=O(1)
\end{aligned}
$$

By Hölder's inequality, (4), and (5),

$$
\begin{aligned}
\sum_{n=2}^{m} n^{k-1}\left|Q_{n 2}\right|^{k} & =\sum_{n=2}^{m+1} n^{k-1}\left|\frac{p_{n}}{P_{n} P_{n-1}} \sum_{\nu=1}^{n-1} \frac{P_{\nu} \epsilon_{\nu}(\nu+1) t_{\nu}^{1}}{\nu(\nu+1)}\right|^{k} \\
& \leq \sum_{n=2}^{m+1} \frac{n^{k-1}}{P_{n-1}}\left(\frac{p_{n}}{P_{n}}\right)^{k}\left[\sum_{\nu=1}^{n-1}\left(\frac{P_{\nu}}{\nu p_{\nu}}\right)^{k} p_{\nu}\left|\epsilon_{\nu}\right|^{k}\left|t_{\nu}^{1}\right|^{k}\right]\left[\frac{1}{P_{n-1}} \sum_{\nu=1}^{n-1} p_{\nu}\right]^{k-1}
\end{aligned}
$$




$$
\begin{aligned}
& =O(1) \sum_{\nu=1}^{m+1}\left(\frac{P_{\nu}}{\nu p_{\nu}}\right)^{k} p_{\nu}\left|\epsilon_{\nu}\right|^{k}\left|t_{\nu}^{1}\right|^{k} \sum_{n=\nu+1}^{m+1} \frac{n^{k-1}}{P_{n-1}}\left(\frac{p_{n}}{P_{n}}\right)^{k} \\
& =O(1) \sum_{\nu=1}^{m+1}\left(\frac{P_{\nu}}{\nu p_{\nu}}\right)^{k} p_{\nu}\left|\epsilon_{\nu}\right|^{k}\left|t_{\nu}^{1}\right|^{k}\left(\frac{\nu^{k-1} p_{\nu}^{k-1}}{P_{\nu}^{k}}\right) \\
& =O(1) \sum_{\nu=1}^{m+1} \nu^{k-1}\left|\epsilon_{\nu}\right|^{k}\left|t_{\nu}^{1}\right|^{k}<\infty .
\end{aligned}
$$

Using Hölder's inequality,(4), and (6),

$$
\begin{aligned}
\sum_{n=2}^{m} n^{k-1}\left|Q_{n 3}\right|^{k} & =\sum_{n=2}^{m+1} n^{k-1}\left|\frac{p_{n}}{P_{n} P_{n-1}} \sum_{\nu=1}^{n-1} P_{\nu} \Delta \epsilon_{\nu} t_{\nu}^{1}\right|^{k} \\
& \leq \sum_{n=2}^{m} \frac{n^{k-1}}{P_{n-1}}\left(\frac{p_{n}}{P_{n}}\right)\left[\sum_{\nu=1}^{n-1}\left(\frac{P_{\nu}}{p_{\nu}}\right)^{k} p_{\nu}\left|\Delta \epsilon_{\nu}\right|^{k}\left|t_{\nu}^{1}\right|^{k}\right]\left[\frac{1}{P_{n-1}} \sum_{\nu=1}^{n-1} p_{\nu}\right]^{k-1} \\
& =O(1) \sum_{\nu=1}^{m}\left(\frac{P_{\nu}}{p_{\nu}}\right)^{k} p_{\nu}\left|\Delta \epsilon_{\nu}\right|^{k}\left|t_{\nu}^{1}\right|^{k} \sum_{n=\nu+1}^{m+1} \frac{n^{k-1}}{P_{n-1}}\left(\frac{p_{n}}{P_{n}}\right)^{k} \\
& =O(1) \sum_{\nu=1}^{m}\left(\frac{P_{\nu}}{p_{\nu}}\right)^{k} p_{\nu}\left|\Delta \epsilon_{\nu}\right|^{k}\left|t_{\nu}^{1}\right|^{k} \frac{\nu^{k-1} p_{\nu}^{k-1}}{P_{\nu}^{k}} \\
& =O(1) \sum_{\nu=1}^{m} \nu^{k-1}\left|\Delta \epsilon_{\nu}\right|^{k}\left|t_{\nu}^{1}\right|^{k}=O(1) .
\end{aligned}
$$

From (5),

$$
\begin{aligned}
\sum_{n=2}^{m} n^{k-1}\left|Q_{n 4}\right|^{k} & =\sum_{n=2}^{m+1} n^{k-1}\left|\frac{p_{n}}{P_{n}} \epsilon_{n} t_{n}^{1}\right|^{k} \\
& =\sum_{n=2}^{m+1} n^{k-1}\left(\frac{p_{n}}{P_{n}}\right)^{k}\left|\epsilon_{n}\right|^{k}\left|t_{n}^{1}\right|^{k} \\
& \leq \sum_{n=2}^{m+1} n^{k-1}\left|\epsilon_{n}\right|^{k}\left|t_{n}^{1}\right|^{k}=O(1) .
\end{aligned}
$$

Part (B). It is sufficient to show that conditions (5) and (6) are satisfied, since any weighted mean matrix satisfying (3) automatically satisfies (4).

Since $\Delta \epsilon_{n}=O(1)\left(n^{-1}\left|\epsilon_{n}\right|\right)$, (6) becomes

$$
\begin{aligned}
\sum_{\nu=1}^{m} \nu^{k-1} O(1)\left(\frac{\left|\epsilon_{\nu}\right|}{\nu}\right)^{k}\left|t_{\nu}^{1}\right|^{k} & =O(1) \sum_{\nu=1}^{m} \frac{1}{\nu^{k}} \nu^{k-1}\left|\epsilon_{\nu}\right|^{k}\left|t_{\nu}^{1}\right|^{k} \\
& \leq O(1) \sum_{\nu=1}^{m} \nu^{k-1}\left|\epsilon_{\nu}\right|^{k}\left|t_{\nu}^{1}\right|^{k}
\end{aligned}
$$


To prove (5),

$$
\begin{aligned}
\sum_{\nu=1}^{n} \nu^{k-1}\left|\epsilon_{\nu}\right|^{k}\left|t_{\nu}^{1}\right|^{k} & =\sum_{\nu=1}^{n} \nu^{k}\left|\epsilon_{\nu}\right|^{k}\left[\sum_{i=1}^{\nu} \frac{1}{i}\left|t_{i}^{1}\right|^{k}-\sum_{i=1}^{\nu-1} \frac{1}{i}\left|t_{i}^{1}\right|^{k}\right] \\
& =n^{k}\left|\epsilon_{n}\right|^{k} \sum_{i=1}^{n} \frac{1}{i}\left|t_{i}^{1}\right|^{k}+\sum_{\nu=1}^{n-1} \sum_{i=1}^{\nu} \frac{1}{i}\left|t_{i}^{1}\right|^{k}\left[\nu^{k}\left|\epsilon_{\nu}\right|^{k}-(\nu+1)^{k}\left|\epsilon_{\nu+1}\right|^{k}\right] \\
& \leq n^{k}\left|\epsilon_{n}\right|^{k} \sum_{i=1}^{n} \frac{1}{i}\left|t_{i}^{1}\right|^{k}+\sum_{\nu=1}^{n} \sum_{i=1}^{\nu} \frac{1}{i}\left|t_{i}^{1}\right|^{k}(\nu+1)^{k} \Delta\left|\epsilon_{\nu}\right|^{k} \\
& =I_{1}+I_{2} .
\end{aligned}
$$

From (7), $I_{1}=O\left(n^{k}\left|\epsilon_{n}\right|^{k} \lambda_{n}^{k}\right)=O(1)$

Since $\Delta\left|\epsilon_{\nu}\right|^{k}=k\left(\left|\epsilon_{\nu}\right|-\left|\epsilon_{\nu+1}\right|\right) \xi^{k-1}$ for some $\xi$ between $\left|\epsilon_{\nu}\right|$ and $\left|\epsilon_{\nu+1}\right|$ by the mean value theorem,

$$
\begin{aligned}
\Delta\left|\epsilon_{\nu}\right|^{k} & \leq k\left|\Delta \epsilon_{\nu}\right| \xi^{k-1} \\
& =O\left(\left|\epsilon_{\nu}\right|^{k-1}\left|\Delta \epsilon_{\nu}\right|\right) \quad \text { since } \quad \epsilon_{n}=o(1) .
\end{aligned}
$$

Therefore

$$
\begin{aligned}
I_{2} & =O(1) \sum_{\nu=1}^{n} \lambda_{\nu}^{k}(\nu+1)^{k} \Delta\left|\epsilon_{\nu}\right|^{k} \\
& =O(1) \sum_{\nu=1}^{n} \lambda_{\nu}^{k}(\nu+1)^{k}\left|\epsilon_{\nu}\right|^{k-1}\left|\Delta \epsilon_{\nu}\right| .
\end{aligned}
$$

Since $\nu \lambda_{\nu}\left|\epsilon_{\nu}\right|=O(1)$,

$$
\begin{aligned}
I_{2}= & O(1) \sum_{\nu=1}^{n} \lambda_{\nu}(\nu+1)\left|\Delta \epsilon_{\nu}\right| \\
= & O(1) \sum_{\nu=1}^{n}\left|\Delta \epsilon_{\nu}\right|\left[\sum_{i=1}^{\nu}(i+1) \lambda_{i}-\sum_{i=1}^{\nu-1}(i+1) \lambda_{i}\right] \\
= & O(1)\left[\left|\Delta \epsilon_{n}\right| \sum_{i=1}^{n}(i+1) \lambda_{i}+\sum_{\nu=1}^{n-1} \sum_{i=1}^{\nu}(i+1) \lambda_{i} \Delta\left(\left|\Delta \epsilon_{\nu}\right|\right)\right] . \\
& \left|\Delta \epsilon_{n}\right| \sum_{i=1}^{n}(i+1) \lambda_{i}=O\left(n^{-1}\left|\epsilon_{n}\right|\right) O\left((n+1) \lambda_{n} n\right) \\
& =O\left((n+1) \lambda_{n}\left|\epsilon_{n}\right|\right)=O(1) .
\end{aligned}
$$

Thus

$$
I_{2} \leq O(1)+\sum_{\nu=1}^{n-1} \sum_{i=1}^{\nu}(i+1) \lambda_{i}\left|\Delta^{2} \epsilon_{\nu}\right|
$$




$$
\begin{aligned}
& \leq O(1)+\sum_{\nu=1}^{n-1} \lambda_{\nu}\left|\Delta^{2} \epsilon_{\nu}\right| \sum_{i=1}^{\nu}(i+1) \\
& =O(1)+O(1) \sum_{\nu=1}^{n-1} \lambda_{\nu} \nu^{2}\left|\Delta^{2} \epsilon_{\nu}\right|=O(1)
\end{aligned}
$$

by $(8)$.

\section{References}

[1] H. Bor, On two summability methods, Math. Proc. Cambrige Phil. Soc. 98(1985), 147-149

[2] T. M. Flett, On an extension of absolute summability and some theorems of Littlewood and Paley, Proc. London Math. Soc. 7(1957), 113-141.

[3] W. T. Sulaiman, A study on a relation between two summability methods, Proc. Amer. Math. Sox. 115(1992), 303-312.

Department of Mathematics, Indiana University, Bloomington, IN 47405-7106.

E-mail: rhoades@indiana.edu

Department of Mathematics, Yüzüncü Yil University, Van, Turkey.

E-mail: ekremsavas@yahoo.com 